\title{
Stress-strain state of the storage silos under the action of the asymmetric load
}

\author{
NataliiaMakhinko ${ }^{1, *}$ \\ ${ }^{1}$ National Aviation University, Kosmonavt Komarov avenue, 1, 03058, Kyiv, Ukraine
}

\begin{abstract}
This paper deals with the investigation of work of the vertical steel cylindrical storage silos for grain with the different thickness of casing sheets and stiffeners at different height sunder the asymmetric wind load. Construction is designed using the analytical method involving decomposition of load into the completed trigonometric series and adding separate stress-strain states of $k$ influence. The construction design model is a thin-walled cylindrical shell reinforced with vertical stiffeners. According to the shell theory, all unknown elements of the stress-strain states from the wind load components are expressed by unknown function $\xi_{k}(x)$. In the analysis, the introduced hypotheses were used, which provides for using exponential dependence to describe the thickness changing law of the casing sheets and stiffeners and the equality of the change rate of these characteristics. The obtained results for silos with corrugated wall showed that the desired function which characterizes the stress-strain state of the construction is determined by ratio of the total cross-section area of all stiffeners to the cross-sectional area of the silo provided this ratio is constant at all heights. Represented calculation formulas for radial, circular and longitudinal displacements, and for strains in cross-sections are simple enough for using in the engineering practice.
\end{abstract}

\section{Introduction}

The main type of load at the vertical cylindrical silos with flexible external frame in the form of a system of vertical stiffeners is vertical and horizontal pressure of a bulk material, which causes axially symmetric loading of the silo, and the pressure of the wind flow, which is an unsymmetrical load and causes the greatest difficulties in determining internal efforts and displacements. Generally, the presence of vertical stiffeners in the silo is considered approximately, by bringing it to the scheme of orthotropic shell, which has different reduced meridional and circular wall thicknesses. For the most of real constructions of this type, the casing sheets and vertical stiffeners are made with different thickness at different heights in order to spare the metal. Considering this feature is causing mathematical difficulties when studying stress-strain state and using the analytical technique. According to the outlined problem, the necessity of proposals development is arising about simplifying analytical procedure of construction calculation.

\footnotetext{
*Correspondingauthor: pasargada1985@gmail.com
} 
The analysis of behavior of the vertical steel silos is a relevant problem for researchers, which is demonstrated by a number of scientific works [1-6], including studies of the authors $[7,8,9]$. It is quite logical that the obtained results of investigation of the stressstrain state of silo constructions are based on works in the shells theory $[10,11,12]$ and verified by calculation of real silo models in the environment of the finite-element analysis. However, because of constantly growing demand to the construction of this type and not always sufficient qualification of the software users, the resulting assessment of work of the structure is often wrong that eventually results in failures, the statistics of which is quite not optimistic now [13, 14]. Therefore analytical (engineering) methods of solving must be definitely used in the practice of calculation of the steel storage silos.

In general, vertical cylindrical storage silo is a thin-walled cylindrical shell reinforced with vertical stiffeners. The finite-element method is most accurate method for defining the full range of internal efforts and displacements of the shell construction. However, investigation of silos with different thicknesses of elements in the finite-element analysis is complicated, since the model formation is multivariable. Accordingly, the results not always correctly reflect the true stress-strain state. This problem especially worsens, when the user doesn't understand the principle of calculation, which leads to the unreasonable choice of the design model and use of the finite-element library. Consequently, authors' proposals are primarily oriented on the development of engineering calculation, and the obtained conclusions will help reach reasonable compromise - to solve the problem with the accuracy sufficient for practical purposes without complicating it with extra calculations.

\section{Research results}

The analytical calculation technique for the vertical cylindrical grain storage silos under the unsymmetrical wind load is based on the possibility of expansion of the aerodynamic coefficient $C_{a e r}$, and correspondingly wind load, into the finite trigonometric series (Fig. 1)

$$
C_{a e r}(\varphi)=a_{0}+a_{1} \cos (\varphi)+a_{2} \cos (2 \varphi)+\ldots+a_{k} \cos (k \varphi)+\ldots+a_{m} \cos (m \varphi),
$$

where $a_{k}$ are coefficients of expansion.

This, accordingly, allows making a calculation to each load element separately. Unknown elements of the stress-strain state under load $W_{k}=A_{k} \cos (k \varphi)$ are defined using the differential equation

$$
\frac{d^{4} \xi_{k}(x)}{d x^{4}}+4 \eta_{w, k}^{4} \xi_{k}(x)=4 \eta_{w, k}^{4} \xi_{k, 0}
$$

where $A_{k}$ is an amplitude value of the $k_{\text {th }}$ element; $\varphi$ is an incidence angle which consider the wind direction; $\eta_{w, k}^{4}$ is the value which consider rigid characteristics of shell in the predetermined conditions of the $k_{\text {th }}$ loading harmonic; $\xi_{k, 0}$ is the proportionality coefficient which has dimension of the function $\xi_{k}(y)$ and considers the character of the harmonious $\operatorname{load} W_{k}$.

The calculation problem of the stress-strain state of the silo with different thickness of the casing sheets $t_{w}(x)$ and vertical stiffeners $t_{p}(x)$ from the mathematical point of view is 
complicated by the dependence of value $\eta_{w, k}$ from longitudinal coordinate $x$ in equation (2). Consequently, this differential equation is considered in the form of

$$
\frac{d^{2}}{d x^{2}}\left[\frac{1}{4 \eta_{\eta, k}^{4}(x)} \frac{d^{2} \xi_{k}(x)}{d x^{2}}\right]+\xi_{k}(x)=\xi_{k, 0} .
$$
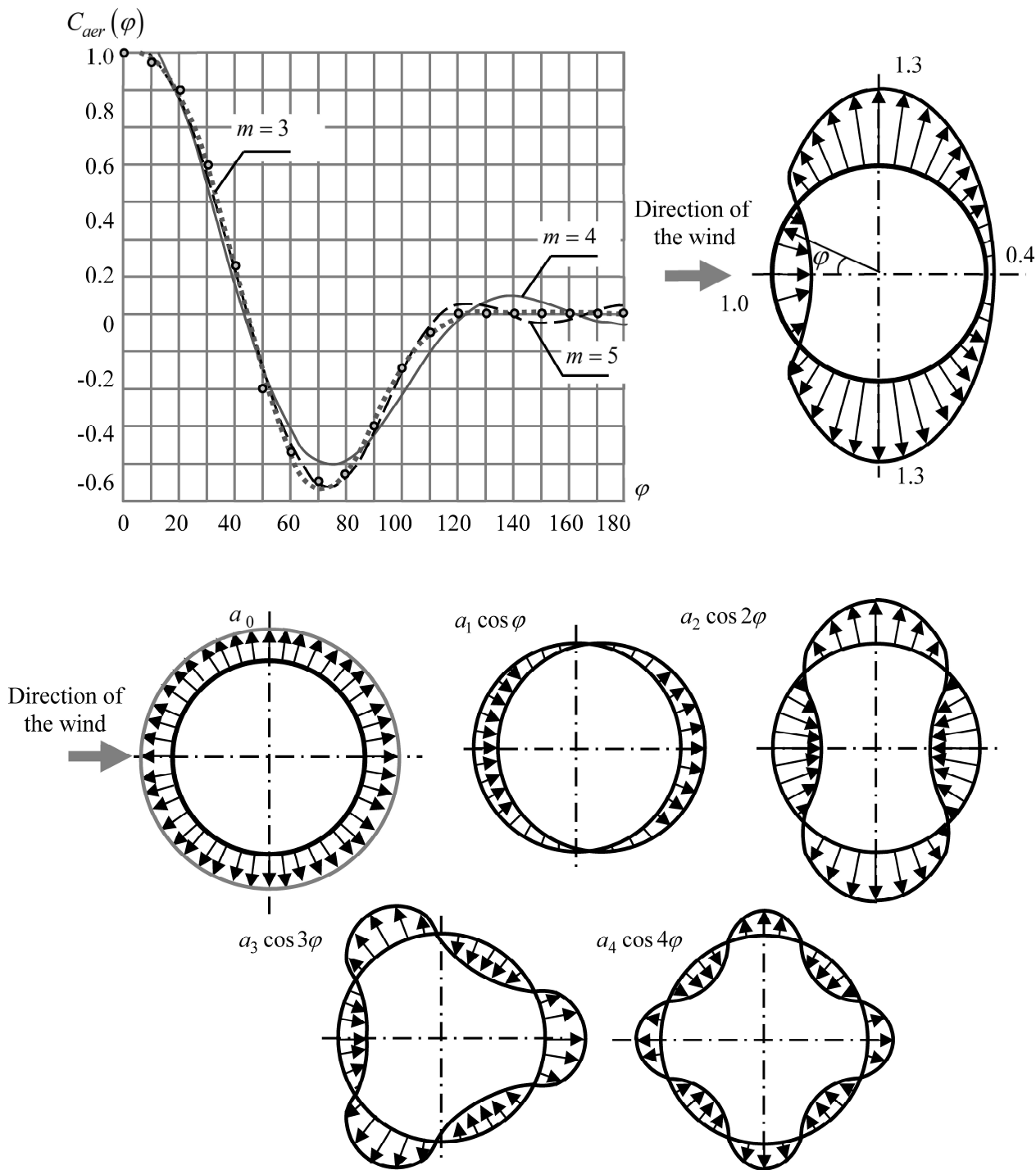

Fig. 1. Series expansion of the aerodynamic coefficient of the wind load around the perimeter of a cylindrical silo.

However, when two hypotheses are formulated which do not contradict the experience of designing storage silos, the problem could be solved in a very simple way. The first hypothesis involves using of exponential dependence for describing the thickness changing law for the casing and stiffeners along the height [9] 


$$
t_{e f}(x)=t_{e f, r} g_{w}(x)=t_{e f, r} \exp \left[-\varepsilon_{w}\left(\frac{x}{H_{w}}\right)\right],
$$

where $H_{w}$ is the height of the storage silo; $t_{e f, r}=t_{w} \ell_{w, 1} / \ell_{w}$ is the thickness of sheets (with the rigidity which is equivalent to the one of the corrugated sheet with the thickness $t_{e f}$ ) which perceive circular efforts; $\ell_{w, 1}$ is the length of the corrugation development with the wave pitch of corrugated sheet of the shell $\ell_{w} ; \varepsilon_{w}$ is a non-dimensional parameter which is responsible for the curve shape $g_{w}(x)$.

According to the second hypothesis, it is supposed that the changing rate of the casing sheets and stiffeners is equal. It means that the same parameters $\varepsilon_{w}$ were used in both dependences. Then after additional expanding of the formula of cylindrical rigidity $D_{r}(x)$, for function $\eta_{w, k}$ we can write

$$
\eta_{w, k}^{4}(x)=\frac{16 k^{4}\left(k^{2}-1\right)^{2}}{\left(1+\alpha_{w p}\right) D_{w}^{6}} t_{w}^{2}(x) \Delta J_{w}(x)
$$

where $\alpha_{w p}=\frac{A_{p} n_{p}}{\pi D_{w} t_{w}}$ is the ratio of the corresponding areas of the stiffeners and the shell; $D_{w}$ is the diameter of the silo; $A_{p}$ is the cross-sectional area; $n_{p}$ is the number of vertical stiffeners; $\Delta J_{w}$ is the ratio of inertia moments of cross-sections of corrugated and flat wall sheets of the silo casing.

Product of $t_{w}^{2}(x) \Delta J_{w}(x)$ is the element which defines dependences of value $\eta_{w, k}$ on $x$. For flat wall silos, ratio $\Delta J_{w}$ is 1 , and for the corrugated wall silo it is expressed through the formula [15]

$$
\begin{gathered}
\Delta J_{w}=\frac{h_{w}^{2} \psi_{2}\left(\delta_{w}\right)}{t_{w}^{2}(x) \delta_{w}} ; \\
\psi_{2}\left(\delta_{w}\right)=\sqrt{32+\delta_{w}^{2}}+2 \sqrt{12+\delta_{w}^{2}}+2.78 \sqrt{1.5+\delta_{w}^{2}},
\end{gathered}
$$

where $\delta_{w}=\ell_{w} / h_{w}$ is the ratio of the period of the sheet profile of the silo wall to its height.

We can see that thicknesses reduce and value $\eta_{w, k}$ becomes independent not only from coordinate $x$, but also from thickness of the casing $t_{w}$. Consequently, there is an important conclusion: for silos with corrugated casing reinforced with vertical stiffeners, the desired function $\xi_{k}(x)$ is not dependent on the absolute thickness value and the law of their change in height, but determined only by the ratio of the total cross-sectional area of all stiffeners to the cross-sectional area of the silo provided this ratio is constant at all heights.

Consequently, according to the general dependences of the shell theory and results, for elements of the stress-strain state of the cylindrical silo with the corrugated wall and different thickness of the casing and stiffeners in different heights, we can write down calculation formulas for radial $w_{k}(y, \varphi)$, circular $v_{k}(y, \varphi)$, longitudinal $u_{k}(y, \varphi)$ displacements and also for strains in cross sections $\sigma_{p, k}(y, \varphi)$ and $\tau_{p, k}(y, \varphi)$ 


$$
\begin{gathered}
w_{k}(y, \varphi)=2 D_{w} \Lambda_{w} \frac{\left[1-\psi_{m, k}(y)\right]}{\left(k^{2}-1\right)^{2}} a_{k} \cos (k \varphi) . \\
v_{k}(y, \varphi)=2 D_{w} \Lambda_{w} \frac{\left[1-\psi_{m, k}(y)\right]}{k\left(k^{2}-1\right)^{2}} a_{k} \sin (k \varphi) . \\
u_{k}(y, \varphi)=\frac{D_{w}}{\Delta_{w}} \Lambda_{w} \frac{\psi_{u, k}(y)}{k^{2}\left(k^{2}-1\right)^{2}} \lambda_{\mu, k} a_{k} \cos (k \varphi) . \\
\sigma_{p, k}(y, \varphi)=\frac{E}{\Delta_{w}^{2}} \Lambda_{w} \frac{\psi_{\sigma, k}(y)}{k^{2}\left(k^{2}-1\right)^{2}} \lambda_{\eta, k}^{2} a_{k} \cos (k \varphi) . \\
\tau_{p, k}(y, \varphi)=\frac{E}{\Delta_{w}^{3}} \Lambda_{w} \frac{\psi_{\tau, k}(y)}{k^{3}\left(k^{2}-1\right)^{2}} \lambda_{\eta, k}^{3} a_{k} \sin (k \varphi),
\end{gathered}
$$

where $\psi_{m, k}(y)$ is calculated according to the formula

$$
\psi_{m, k}(y)=M_{1, k} \cosh \left(y \lambda_{\eta, k}\right) \sin \left(y \lambda_{\eta, k}\right)+\cos \left(y \lambda_{\eta, k}\right)\left[M_{2, k} \exp \left(-y \lambda_{\eta, k}\right)+M_{3, k} \exp \left(y \lambda_{\eta, k}\right)\right] .
$$

$M_{1, k}, M_{2, k}$ and $M_{3, k}$ are non-dimensional functions of response of the silo; functions $\psi_{u, k}(y), \quad \psi_{\sigma, k}(y)$ and $\psi_{\tau, k}(y)$ are similar according to the mathematic nature of $\psi_{m, k}(y)$ and determined by expressions of the first, second and third derivatives; $E$ is the modulus of the material resilience.

Special attention must be paid to compliance coefficient $\Lambda_{w}$ of the silo which is the proportionality coefficient in formulas (7)-(11) and is the function of a non-dimension height $y=x / H_{w}$. Due to the received dependence (4) for thicknesses of the casing $t_{w}(x)$ and stiffeners $t_{p}(x)$, we will have for the confluence (in this case the origin is considered to be at the top of the silo)

$$
\Lambda_{w}(y)=\frac{3 \delta_{D t}^{6}\left(1-\mu^{2}\right)}{8 \Delta J_{w}} \frac{w_{p}}{E} \exp \left[\varepsilon_{w}(1-y)\right]
$$

where all parameters which include wall thickness $t_{w}$ and stiffener area $A_{p}$, refer to the confinement area of the silo.

For flat wall silos, there is no such a simplifying procedure and the solution of equation (3) must be searched according to the rules of differential calculation. But if the flat sheet considers as the borderline case of the corrugated one, then all formulated above conclusions will remain in force. In fact, corrugating parameters can always be selected so "bad" to provide equality of bending rigidity to the flat sheet.

Accordingly, formula (13) could be extended to the flat wall silos without considering lengthy solution of the differential equality. Acceptability of such idealization was verified at some finite-element models, which confirm the assumption. Results are also confirmed by conclusions of study [15], where the main differential equation of the stress-strain state of the silo with different thickness under the axially symmetric load was solved. 


\section{Conclusions}

1. All unknown elements of the stress-strain state from the wind load elements can be expressed through function $\xi_{k}$ which satisfies the linear differential equation (1).

2. Introduced hypotheses to describe the thickness changing law for the casing sheets and vertical stiffeners along the height and equality of changing rate of these thicknesses allow simplifying the problem of location of strains in cross-sections and the radial, circular and longitudinal displacements, and also allow using easy calculation formulas.

3. For silos with corrugated casing reinforced with vertical stiffeners, the desired function is not dependent on the absolute thickness value and the change law along the height, and is determined only by the ratio of the total cross-section area of all stiffeners to the cross-sectional area of the silo provided this ratio is constant at all heights.

4. For flat wall containers the obtained results are true, only if the flat sheet is considered as the borderline case of the corrugated one.

\section{References}

1. M. Wojcik, P.Iwicki, J. Tejchman, Thin-Walled Str.49, 8 947-963 (2011).

2. A. Sadowski, M. Rotter,Thin-Walled Str.61 169-179 (2012).

3. Y. Zhao, Q. Cao, L. Su,Thin-Walled Str.73 337-349 (2013).

4. R. Stepanov, D. Romensky, V. Mushchanov, Science9, 6152-160 (2017).

5. C. Maraveas, G. Balokas, K. Tsavdaridis, Thin-Walled Str.95152-160 (2015).

6. S. Maleki, A. Mehretehran, Engineering Failure Analysis90 156-167 (2018).

7. A. Makhinko, N. Makhinko,Pidvodni tekhnolohii06 16-27 (2017).

8. N. Makhinko, Visnyk ODABA7068-74, (2018).

9. A. Makhinko, N. Makhinko, Industrial Machine Building69-78 (2018).

10. V.Vlasov,Obshchaia teoriia obolochek(Akademiia nauk, Moskva, 1962).

11. S. Kahn,Stroitelnaia mekhanika obolochek(Mashinostroenie, Moskva, 1966).

12. A. Goldenveizer,Teoriia uprugikh tonkikh obolochek (Nauka, Moskva, 1976).

13. M. Kazakevich, D. Bannikov,Metallicheskie konstruktsii1 59-66 (2002).

14. A. Raeesi, H. Ghaednia, J. Zohrehheydariha, S. Das, Engineering Failure Analysis79 749-761 (2017).

15. O. Lapenko, N. Makhinko,Nauka i budivnytstvo,2(16) 40-45 (2018). 